\title{
Translating Improvements with Ixekizumab in Clinical Trial Outcomes into Clinical Practice: ASAS40, Pain, Fatigue, and Sleep in Ankylosing Spondylitis
}

\author{
Philip Mease · Jessica A. Walsh - Xenofon Baraliakos · Robert Inman • \\ Kurt de Vlam • James Cheng-Chung Wei · Theresa Hunter • Gaia Gallo • \\ David Sandoval $\cdot$ Fangyi Zhao $\cdot$ Yan Dong $\cdot$ Rebecca Bolce $\cdot$ Helena Marzo-Ortega
}

Received: May 3, 2019 / Published online: June 28, 2019

(c) The Author(s) 2019

\section{ABSTRACT}

Introduction: Ixekizumab, a humanized interleukin-17A antibody, has shown efficacy in ankylosing spondylitis (AS), with a greater proportion of ixekizumab-treated patients achieving an ASAS40 (Assessment of Spondyloarthritis International Society 40) endpoint compared to placebo. An ASAS40 response is a high standard

Enhanced Digital Features To view enhanced digital features for this article go to: https://doi.org/10.6084/ m9.figshare.8263097.

P. Mease $(\square)$

Division of Rheumatology Clinical Research, University of Washington and Swedish Medical Center, Seattle, WA, USA

e-mail: pmease@philipmease.com

\section{J. A. Walsh}

University of Utah School of Medicine and Salt Lake City Veterans Affairs Medical Center, Salt Lake City, UT, USA

\section{Baraliakos}

Rheumazentrum Ruhrgebiet Herne, Ruhr-

University, Bochum, Germany

R. Inman

Departments of Immunology and Medicine,

University Health Network, University of Toronto,

Toronto, ON, Canada

K. de Vlam

Division of Rheumatology, University Hospitals

Leuven, Leuven, Belgium that is not routinely used in clinical practice. The goals of this study were (a) to measure improvement in ixekizumab-treated patients in the four ASAS treatment response domains and in other patient-reported outcomes, and (b) to determine how the ASAS response was associated with changes in spinal pain at night, fatigue, sleep, and the Short Form 36-Item Physical Component Summary (SF-36 PCS).

Methods: The COAST-V and COAST-W trials were randomized, double-blind, controlled trials examining ixekizumab efficacy in patients with AS who were biologic disease-modifying

\section{K. de Vlam}

Skeletal Biology and Engineering Research Center, Department of Development and Regeneration, KU Leuven, Leuven, Belgium

J. C.-C. Wei Institute of Medicine, Chung Shan Medical University, Taichung, Taiwan

J. C.-C. Wei Department of Medicine, Chung Shan Medical University Hospital, Taichung, Taiwan

J. C.-C. Wei

Graduate Institute of Integrated Medicine, China Medical University, Taichung, Taiwan

T. Hunter - G. Gallo · D. Sandoval - F. Zhao .

Y. Dong · R. Bolce

Eli Lilly and Company, Indianapolis, IN, USA 
antirheumatic drug (bDMARD)-naïve and tumor necrosis factor inhibitor (TNFi)-experienced, respectively. Data for the ASAS treatment response domains and other outcomes were collected through 16 weeks. Comparisons between treatment groups were made using a mixed-effects model for repeated measures. To determine how the ASAS response was associated with the changes in spinal pain at night, fatigue, sleep, and SF-36 PCS, comparisons were made between patient groups according to their level of treatment response (ASAS40 vs. ASAS20 vs. ASAS20 nonresponse) using analysis of covariance.

Results: Compared with placebo, patients treated with ixekizumab reported significantly greater improvement in the four ASAS treatment response domains and other outcomes $(p<0.05)$. Results were consistent for bDMARD-naïve and TNFi-experienced patients. Compared to ASAS20 nonresponders, patients who achieved ASAS40 reported significantly greater mean changes in spinal pain at night (1.0 vs. 5.1 for bDMARD-naïve; 0.5 vs. 5.4 for TNFi-experienced), fatigue (0.6 vs. 3.8 for bDMARD-naïve; 0.2 vs. 3.9 for TNFi-experienced), sleep quality (1.1 vs. 4.0 for bDMARDnaïve; 0.8 vs. 4.9 for TNFi-experienced), and SF36 PCS (2.6 vs. 11.6 for bDMARD-naïve; 1.2 vs. 12.6 for TNFi-experienced) $(p<0.0001)$.

Conclusion: Patients with AS who were treated with ixekizumab reported greater improvements in multiple patient-reported outcomes than patients who received placebo. Importantly, achieving ASAS40 was associated with a 2.6-fold to 5.3-fold greater improvement in pain, fatigue, sleep, and quality of life for bDMARD-naïve patients, and a 5.1-fold to 18.5fold greater improvement for TNFi-experienced patients, compared to ASAS20 nonresponders.

Trial Registration: ClinicalTrials.gov identifiers: NCT02696785 and NCT02696798.

Funding: Eli Lilly and Company.

H. Marzo-Ortega

NIHR Leeds Biomedical Research Centre, Leeds

Teaching Hospitals Trust and Leeds Institute of

Rheumatic and Musculoskeletal Medicine,

University of Leeds, Leeds, UK
Keywords: Ankylosing spondylitis; ASAS40; Axial spondyloarthritis; Fatigue; Inflammation; Ixekizumab; Pain; Sleep

\section{INTRODUCTION}

Ankylosing spondylitis (AS), or radiographic axial spondyloarthritis, is a potentially debilitating inflammatory disease of the sacroiliac joint and spine that impacts $0.5-1 \%$ of the population $[1,2]$. Patients with AS show evidence of sacroiliitis on plain films of the sacroiliac joints according to specific criteria [3]. These disease characteristics are important for the diagnosis of AS. However, there are many other facets of AS that affect patients profoundly. Patients suffer with pain, fatigue, and stiffness, plus physical limitations, all of which impact patients' quality of life (QoL) [4]. Several types of therapy are recommended, including exercise, physical therapy, nonsteroidal antiinflammatory drugs, and biologic disease-modifying antirheumatic drugs (bDMARDs) [5]. While tumor necrosis factor inhibitors (TNFi) improve symptoms for many patients, up to $40 \%$ of patients with AS do not respond well or sustain a response to these medications [6-10].

Several new bDMARDs are in development or approved, including those that bind interleukin 17 (IL-17), a key cytokine in the pathogenesis of AS [11]. Ixekizumab is a humanized immunoglobulin G4 monoclonal antibody which targets IL-17A, one of the six members of the IL-17 family [12]. The results of two recently completed clinical trials demonstrated ixekizumab's efficacy in treating patients with AS: COAST-V tested ixekizumab in patients who had never been treated with a bDMARD (bDMARD-naïve), and COAST-W tested ixekizumab in patients who had not responded to or had been intolerant of one or two TNFis (TNFiexperienced) [13, 14].

A commonly used measure of AS treatment efficacy in clinical trials is the Assessment of Spondyloarthritis International Society response criteria, including ASAS20, ASAS40, and ASAS partial remission [15]. Four domains are used for the ASAS clinical trial treatment response assessments: patient global (patient 
global assessment of disease), spinal pain, function (as assessed by the Bath Ankylosing Spondylitis Functional Index [BASFI]), and inflammation (stiffness) [15]. The ASAS treatment response domains rely on patient-reported outcomes, highlighting the importance of a patient's own health assessment for evaluating the efficacy of AS treatments.

Historically, clinical trials for bDMARDs have used ASAS20 as the primary endpoint for determining efficacy in AS [16-19]. The COAST$\mathrm{V}$ and COAST-W trials of ixekizumab efficacy in AS used ASAS40, a higher standard, as the primary endpoint. COAST-V and COAST-W reported statistically significantly higher proportions of patients achieving ASAS40 in ixekizumab treatment groups versus placebo $[13,14]$. Although the ASAS response is of great value in the context of clinical trials, in daily clinical practice, physicians focus on each individual's clinical symptoms rather than calculating an ASAS response. Thus, this report seeks to translate the clinical impact of achieving an ASAS40 response into the individual patient-reported outcomes utilized in clinical practice. The goals of this study were (a) to measure improvement in ixekizumab-treated patients both in the ASAS response criteria domains and in other outcomes, and (b) to quantify the incremental improvement in patient-reported outcomes for patients who achieve ASAS40 compared to those who did not achieve ASAS20.

\section{METHODS}

\section{Trial Design}

The COAST-V and COAST-W trial designs have been published previously [13, 14]. Briefly, COAST-V and COAST-W were phase 3 , multicenter, randomized, double-blind, placebocontrolled trials. COAST-V contained an activereference arm, in which patients received adalimumab [14]. COAST-V and COAST-W were conducted at 84 and 106 sites in 12 and 15 countries, respectively, across North America, South America, Europe, and Asia. Each study was approved by ethical review boards at each site before the start of the studies. COAST-V and COAST-W were registered on ClinicalTrials.gov as NCT02696785 and NCT02696798, respectively. All procedures performed in these studies involving human participants were in accordance with the ethical standards of the institutional and/or national research committees at all sites where these studies were conducted and with the 1964 Declaration of Helsinki and its later amendments or comparable ethical standards. The master ethics committee was Schulman Associates IRB, Cincinnati, OH, USA. The full lists of investigators and sites are provided in the primary manuscript supplements $[13,14]$. Informed consent was obtained from all individual participants included in the studies.

\section{Trial Participants}

COAST-V and COAST-W included patients who fulfilled the ASAS criteria for radiographic axial spondyloarthritis [13-15]. Post hoc analysis showed that these patients also fulfilled the modified New York criteria for AS [20]. Patients in COAST-V and COAST-W had long-standing symptoms of AS for a mean (standard deviation, SD) of 16.0 (10.3) and 18.4 (11.1) years, and had been diagnosed $7.7(8.4)$ and 11.6 (9.1) years prior to the study, respectively [13, 14]. Mean (SD) baseline Ankylosing Spondylitis Disease Activity Scores (ASDAS) were 3.8 (0.8) for COAST-V patients and 4.1 (0.8) for COAST-W patients [13, 14], which indicates very high disease activity in the populations included in the trials (ASDAS > 3.5) [21]. Patients had mean (SD) baseline C-reactive protein (CRP) scores of 13.5 (17.1) and 17.8 (26.6) $\mathrm{mg} / \mathrm{L}$, with 64\% and $66 \%$ having elevated CRP at baseline (CRP > $5 \mathrm{mg} / \mathrm{L}$ ), for COAST-V and COAST-W, respectively $[13,14]$. COAST-V patients had no history of biologic or other immunomodulatory agents, including investigational therapies (bDMARD-naïve) [14]. COAST-W patients had prior treatment with one or two TNFi and had discontinued at least one TNFi due to inadequate response or intolerance (TNFi-experienced) [13]. A majority of the TNFi-experienced 
patients $(89.9 \%)$ had responded inadequately to at least one TNFi.

\section{Randomization and Blinding}

In COAST-V, 341 patients were randomized 1:1:1:1 to receive subcutaneous injections of $80 \mathrm{mg}$ ixekizumab every 2 weeks (Q2W, $n=83)$, $80 \mathrm{mg}$ ixekizumab every 4 weeks $(\mathrm{Q} 4 \mathrm{~W} ; n=81)$, $40 \mathrm{mg}$ adalimumab Q2W $(n=90)$, or placebo Q2W $(n=87)$ [14]. In COAST-W, 316 patients were randomized 1:1:1 to receive subcutaneous injections of $80 \mathrm{mg}$ ixekizumab Q2W $(n=98)$, $80 \mathrm{mg}$ ixekizumab Q4W $(n=114)$, or placebo Q2W $(n=104)$ [13]. Patients receiving ixekizumab in both trials were randomized 1:1 to receive a starting dose of either $80 \mathrm{mg}$ or $160 \mathrm{mg}$ ixekizumab at week $0[13,14]$.

\section{Assessments}

An ASAS40 response was the primary endpoint for both COAST-V and COAST-W. ASAS40 is defined as $\mathrm{a} \geq 40 \%$ improvement and an absolute improvement from baseline of $\geq 2$ units (range $0-10$ ) in $\geq 3$ of the 4 domains without any worsening in the remaining domain [15]. Secondary endpoints included ASAS20 responses and changes from baseline in Bath Ankylosing Spondylitis Disease Activity Index (BASDAI) scores, BASFI scores, and the Medical Outcomes Study 36-Item Short-Form Health Survey Physical Component Summary version 2 (SF-36 PCS). An ASAS20 response is defined as $\geq 20 \%$ improvement and an absolute improvement from baseline of $\geq 1$ units (range 0-10) in $\geq 3$ of the 4 domains and no worsening of $\geq 20 \%$ and $\geq 1$ unit (range 0-10) in the remaining domain [15]. Both BASDAI question 1 (Q1) and a second independently developed measure were used to measure fatigue. The second measure, the Fatigue Numeric Rating Scale (NRS), assessed the patient's level of fatigue in the last $24 \mathrm{~h}$ from 0 (no fatigue) to 10 (as bad as you can imagine) [22]. Pain was assessed as described in the ASAS Handbook [15], asking patients the following two questions about their pain in the last week: (1) "How much pain of your spine due to ankylosing spondylitis do you have?" and (2) "How much pain of your spine due to ankylosing spondylitis do you have at night?" The answers to the two pain questions were recorded and a rating of between 0 (no pain) and 10 (most severe pain) was assigned. Results from the first pain question were used to derive responses for the calculation of pain in ASAS20 and ASAS40. Patient global, BASFI, and inflammation (the average of BASDAI Q5 [intensity of morning stiffness] and Q6 [duration of morning stiffness]) scores were used to calculate the other three domains for ASAS20 and ASAS40 [15]. Each domain received a score from 0 to 10 . Unless indicated otherwise, data were collected at each study visit (baseline and weeks $1,2,4,8,12$, and 16). SF-36 PCS data were collected at baseline and weeks 4, 8, and 16 . Fatigue NRS and Jenkins Sleep Evaluation Questionnaire (JSEQ) data were collected at baseline and weeks 8 and 16 [23]. Higher JSEQ scores indicate an increased frequency of disrupted sleep [23].

\section{Statistical Analyses}

For comparisons between treatment groups, changes from baseline for the ASAS treatment response domains (patient global, spinal pain, BASFI, inflammation [BASDAI Q5 and Q6]) and other patient-reported outcomes (spinal pain at night, fatigue [BASDAI Q1 and NRS], JSEQ, BASDAI, and SF-36 PCS) were analyzed using a mixed-effects model for repeated measures. No statistical comparisons were performed between adalimumab and ixekizumab because the adalimumab was included as an active reference. Post hoc association analyses between ASAS response status and change in patient-reported outcome measures were conducted by pooling all patients (placebo and adalimumab groups included) into three groups: patients who did not achieve ASAS20 (ASAS20 nonresponders), patients who achieved ASAS20 but not ASAS40, and patients who achieved ASAS40. Data from the two trials were kept separate in this analysis in order to determine whether there were differences in responses between bDMARD-naïve and TNFi-experienced patients. Changes from baseline in pain, fatigue (NRS), JSEQ, and SF-36 
PCS scores were compared between ASAS response groups using analysis of covariance (ANCOVA) after adjusting for baseline values, age, and gender. Post hoc comparisons were conducted with Scheffe's correction. Missing data were imputed using nonresponder imputation for all categorical endpoints and modified baseline observation carried forward for the ANCOVA model. Statistical analyses were performed using SAS version 9.2 or higher. Nominal $p$ values are shown.

\section{RESULTS}

Baseline disease data for the bDMARD-naïve and TNFi-experienced patients are shown in Table 1. In general, the TNFi-experienced patients had numerically higher levels for most measures, which was indicative of them having more severe disease at baseline compared with bDMARD-naïve patients.

\section{Improvement in Pain, Fatigue, Inflammation, Sleep, and Quality of Life with Ixekizumab}

To determine the effect of ixekizumab specifically on the ASAS treatment response domains, the outcomes of the four domains were compared. Compared with placebo, patients treated with ixekizumab had greater improvements in patient global (Fig. 1a, b), spinal pain (Fig. 1c, d), inflammation (Fig. 1e, f), and function (BASFI) (Fig. 1g; [13]) throughout the 16 weeks assessed (Table 2; Fig. 1, $p<0.01$ ). The data for function in TNFi-experienced patients have been published previously [13]. Patients who were bDMARD-naïve and treated with the active reference adalimumab also showed significant improvements in the ASAS treatment response domains when compared with placebo. Improvements with ixekizumab treatment in the ASAS treatment response domains were consistent between the bDMARD-naïve and TNFi-experienced patients, with significant improvements reported as early as week 1 $(p<0.01)$ [13].

Patient-reported outcomes outside of the ASAS treatment response domains were also
Table 1 Baseline disease characteristics for bDMARDnaive and TNFi-experienced patients in COAST-V and COAST-W

\begin{tabular}{|c|c|c|}
\hline & $\begin{array}{l}\text { COAST V: } \\
\text { bDMARD- } \\
\text { naïve } \\
N=341\end{array}$ & $\begin{array}{l}\text { COAST-W: } \\
\text { TNFi- } \\
\text { experienced } \\
N=316\end{array}$ \\
\hline \multicolumn{3}{|l|}{$\begin{array}{l}\text { ASAS treatment } \\
\text { response } \\
\text { domains }\end{array}$} \\
\hline Patient global & $7.0(1.6)$ & $7.9(1.7)$ \\
\hline Spinal pain & $7.2(1.5)$ & $7.9(1.5)$ \\
\hline $\begin{array}{l}\text { Inflammation } \\
\text { (BASDAI Q5/ } \\
\text { Q6) }\end{array}$ & $6.7(1.7)$ & $7.3(1.7)$ \\
\hline BASFI & $6.2(2.0)$ & $7.3(1.7)$ \\
\hline \multicolumn{3}{|l|}{ Other outcomes } \\
\hline $\begin{array}{l}\text { Spinal pain at } \\
\text { night }\end{array}$ & $7.0(1.6)$ & $7.7(1.7)$ \\
\hline $\begin{array}{l}\text { Fatigue (BASDAI } \\
\text { Q1) }\end{array}$ & $7.1(1.6)$ & $7.7(1.4)$ \\
\hline Fatigue (NRS) & $6.8(1.7)$ & $7.3(1.8)$ \\
\hline JSEQ & $8.2(5.2)$ & $10.4(5.6)$ \\
\hline BASDAI & $6.7(1.4)$ & $7.4(1.3)$ \\
\hline SF-36 PCS & $33.4(8.0)$ & $28.6(7.9)$ \\
\hline
\end{tabular}

Data shown as mean (SD)

$A S A S$ Assessment of Spondyloarthritis International Society, BASDAI Bath Ankylosing Spondylitis Disease Activity Index, $B A S F I$ Bath Ankylosing Spondylitis Functional Index, $b D M A R D$ biologic disease-modifying antirheumatic drugs, JSEQ Jenkins Sleep Evaluation Questionnaire, NRS Numeric Rating Scale, SF-36 PCS Medical Outcomes Study 36-Item Short-Form Health Survey Physical Component Summary, SD standard deviation, TNFi tumor necrosis factor inhibitor

analyzed. Compared with placebo, patients treated with ixekizumab reported greater improvements from baseline for both spinal pain at night (Fig. 2a, b; Table 2; $p<0.001$ ) and fatigue assessed using BASDAI Q1 (Fig. 2c, d; 
Table $2 ; p<0.05)$. These significant improvements with ixekizumab treatment were seen as early as week 1 and remained higher than placebo through at least week 16 (Fig. 2; Table 2; $p<0.05)$. The results were consistent between the bDMARD-naïve patients and the TNFi-experienced patients (Fig. 2; Table 2). Patients also reported greater improvements in fatigue at weeks 8 and 16 when measured by the Fatigue NRS [22] (Fig. 2e, f; Table 2; $p<0.05$ ). In bDMARD-naïve patients, there was a significant improvement in sleep quality at weeks 8 and 16 for patients treated with ixekizumab Q2W, and at week 8 for patients treated with ixekizumab Q4W (Fig. 2g; Table 2; $p<0.05$ ). In the TNFiexperienced population, numerical improvements in sleep quality were observed for both ixekizumab dosing regimens, and a significant improvement was observed in patients treated with ixekizumab Q4W at week 8 (Fig. 2h; Table 2; $p<0.05$ ).

To determine the impact of ixekizumab on overall disease activity, improvements in BASDAI were compared between the treatment arms. In bDMARD-naïve patients, patients reported significantly greater improvements in BASDAI scores with ixekizumab treatment than placebo (Fig. 3a; Table 2; $p<0.01$ ). The data for BASDAI in TNFi-experienced patients have been published previously [13]. The impact of ixekizumab treatment on health-related QoL was assessed using SF-36 PCS scores. Patients who were bDMARD-naïve reported greater improvements in health-related QoL when treated with ixekizumab, with significant differences observed versus placebo starting at week 4 , the first time SF-36 PCS was measured after baseline, and continuing to week 16 (Fig. $3 b, p<0.01$ ). The data for SF-36 PCS in TNFi-experienced patients have been published previously [13].

\section{ASAS40 is Associated with Reduced Pain, Fatigue, and Sleep, and Improved QoL}

The data presented thus far indicate that significant improvements were observed in the four ASAS treatment response domains plus spinal pain at night, fatigue, QoL, and, to a lesser degree, sleep quality. However, the
Fig. 1 Least-squares mean (LSM) changes from baseline in the ASAS treatment response domains of $\mathbf{a}, \mathbf{b}$ patient global, $\mathbf{c}, \mathbf{d}$ spinal pain, e, $\mathbf{f}$ inflammation (BASDAI Q5 and Q6), and $\mathbf{g}$ function (BASFI) for $\mathbf{a}, \mathbf{c}, \mathbf{e}, \mathbf{g}$ bDMARDnaïve patients (COAST-V) and $\mathbf{b}, \mathbf{d}, \mathbf{f}$ TNFi-experienced patients (COAST-W). Comparisons with $\mathrm{PBO}$ were made using a mixed-effects model for repeated measures. ${ }^{* * *} p<0.001$ vs. $\mathrm{PBO} ;{ }^{* *} p<0.01$ vs. PBO. The BASFI data for TNFi-experienced patients (COAST-W) were published previously [13]. ADA adalimumab, ASAS Assessment of Spondyloarthritis International Society, $B A S D A I$ Bath Ankylosing Spondylitis Disease Activity Index, BASFI Bath Ankylosing Spondylitis Functional Index, $b D M A R D$ biologic disease-modifying antirheumatic drugs, $I X E$ ixekizumab, $P B O$ placebo, $Q 2 W$, every 2 weeks, $Q 4 W$ every 4 weeks, $T N F i$ tumor necrosis factor inhibitor

primary endpoint in these studies was achievement of ASAS40, a composite measure not routinely used in clinical practice. To assess the relative benefit of achieving different levels of ASAS response, data for all study arms were pooled and then divided into three groups: ASAS20 nonresponders, patients achieving ASAS20 but not ASAS40, and ASAS40 responders. Because the measures for spinal pain at night, fatigue (NRS), sleep quality, and SF-36 PCS were not used to derive ASAS40, these patient-reported outcomes were compared between the three response groups. As expected, the greatest improvements at week 16 were observed in the patients who achieved ASAS40. When compared to the ASAS20 nonresponders, bDMARD-naïve patients who achieved ASAS40 had significantly greater improvements from baseline for spinal pain at night (1.0 vs. 5.1, $p<0.0001)$, fatigue (0.6 vs. 3.8, $p<0.0001)$, sleep quality (1.1 vs. $4.0, p<0.0001)$, and SF-36 PCS (2.6 vs. 11.6, $p<0.0001$ ) (Fig. 4). These results corresponded to a 4.1-fold improvement in spinal pain at night, a 5.3-fold improvement for fatigue, a 2.6-fold improvement in sleep quality, and a 3.5-fold improvement in healthrelated QoL. Interestingly, the benefit of achieving an ASAS40 response occurred irrespective of previous exposure to bDMARDs. Compared with ASAS20 nonresponders, TNFiexperienced patients with AS who achieved ASAS40 also had significantly greater 


\section{bDMARD-naïve}

Week
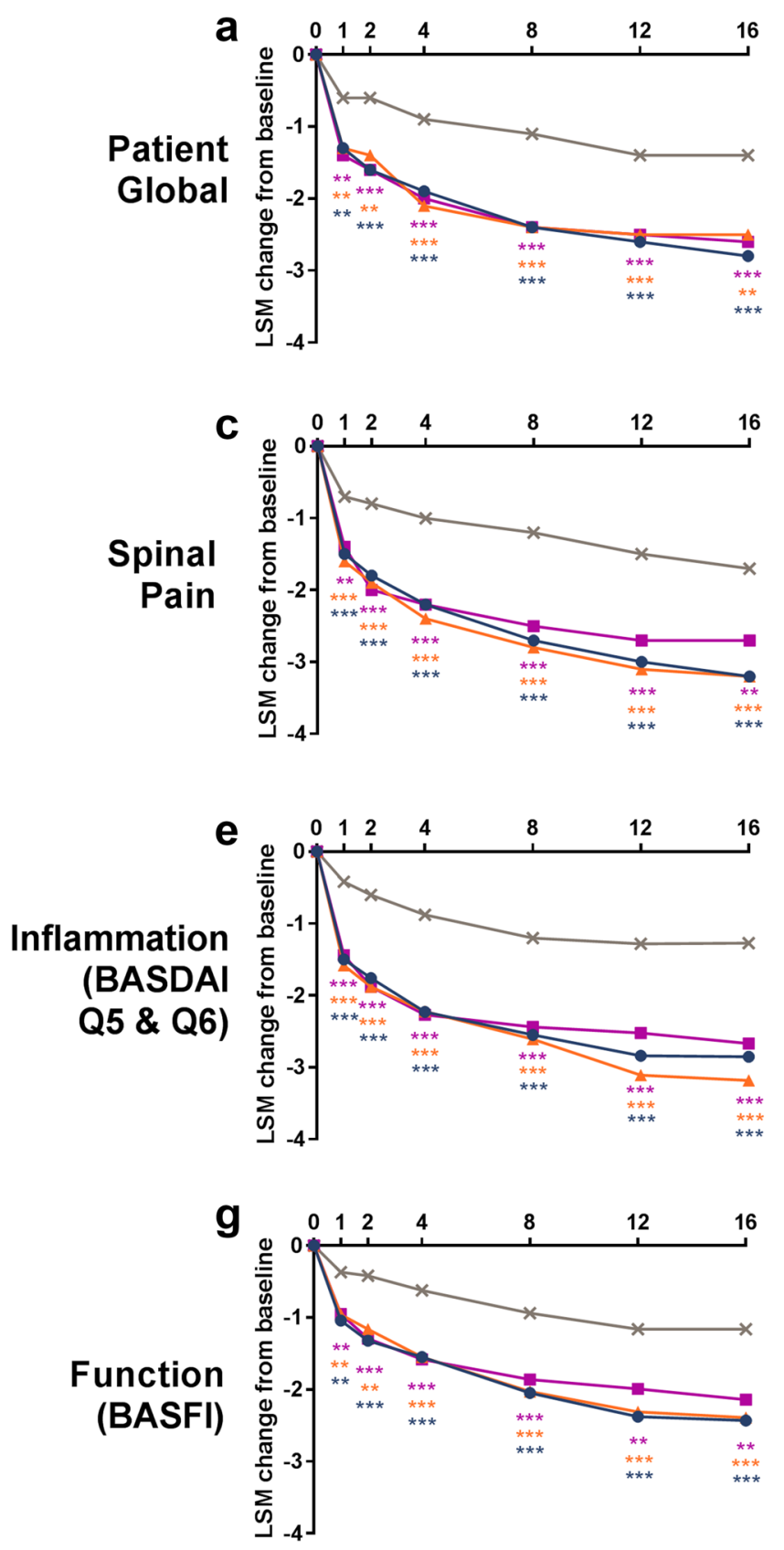

$\multimap I X E Q 2 W \_I X E Q 4 W \rightarrow A D A * P B O$

b
TNFi-experienced
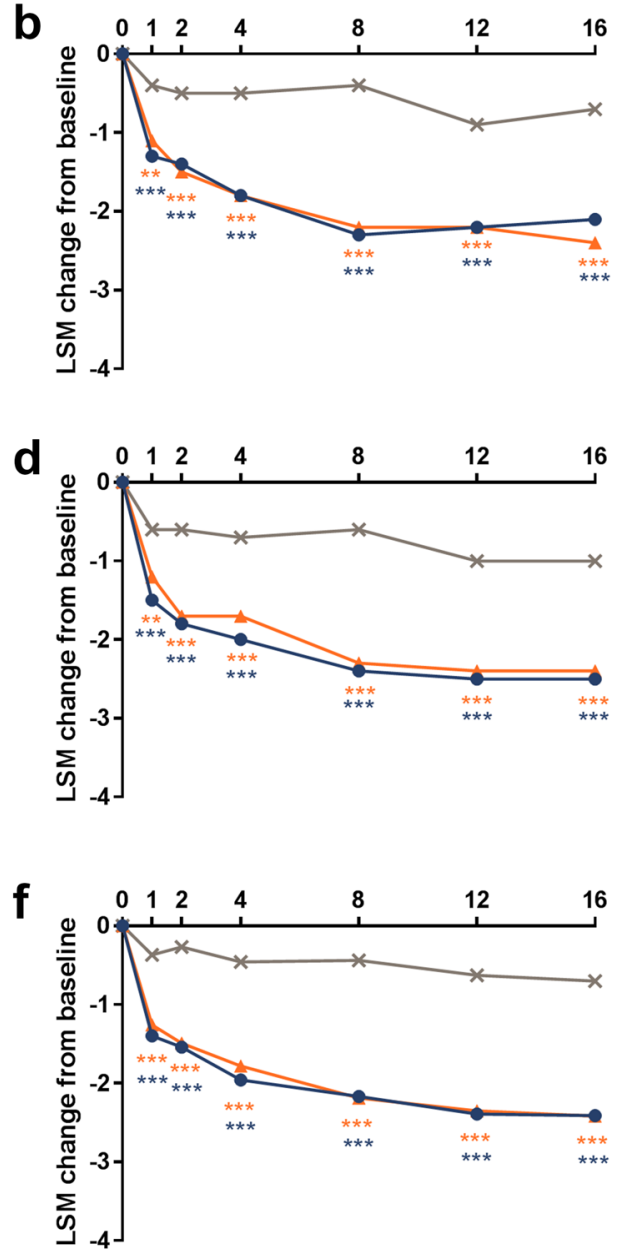

- IXE Q2W - IXE Q4W -1- ADA $*$ PBO 
Table 2 Changes from baseline in patient-reported outcomes for bDMARD-naïve (COAST-V) and TNFi-experienced patients (COAST-W)

\begin{tabular}{|c|c|c|c|c|c|}
\hline \multirow[t]{2}{*}{ Study } & \multicolumn{5}{|c|}{ Least-squares mean (standard error) } \\
\hline & Week & IXE Q2W & IXE Q4W & ADA & PBO \\
\hline \multicolumn{6}{|c|}{ ASAS treatment response domains } \\
\hline \multicolumn{6}{|l|}{ Patient global } \\
\hline \multirow[t]{2}{*}{ COAST-V } & 8 & $-2.4(0.24)^{*}$ & $-2.4(0.24)^{*}$ & $-2.4(0.23)^{*}$ & $-1.1(0.24)$ \\
\hline & 16 & $-2.8(0.25)^{*}$ & $-2.5(0.25)^{\gamma}$ & $-2.6(0.24)^{*}$ & $-1.4(0.24)$ \\
\hline \multirow[t]{2}{*}{ COAST-W } & 8 & $-2.3(0.22)^{*}$ & $-2.2(0.20)^{*}$ & - & $-0.4(0.21)$ \\
\hline & 16 & $-2.1(0.24)^{*}$ & $-2.4(0.22)^{*}$ & - & $-0.7(0.23)$ \\
\hline \multicolumn{6}{|l|}{ Spinal pain } \\
\hline \multirow[t]{2}{*}{ COAST-V } & 8 & $-2.7(0.23)^{*}$ & $-2.8(0.24)^{*}$ & $-2.5(0.23)^{*}$ & $-1.2(0.23)$ \\
\hline & 16 & $-3.2(0.24)^{*}$ & $-3.2(0.25)^{*}$ & $-2.7(0.23)^{\gamma}$ & $-1.7(0.24)$ \\
\hline \multirow[t]{2}{*}{ COAST-W } & 8 & $-2.4(0.23)^{*}$ & $-2.3(0.20)^{*}$ & - & $-0.6(0.21)$ \\
\hline & 16 & $-2.5(0.25)^{*}$ & $-2.4(0.23)^{*}$ & - & $-1.0(0.24)$ \\
\hline \multicolumn{6}{|c|}{ Inflammation (BASDAI Q5 and Q6) } \\
\hline \multirow[t]{2}{*}{ COAST-V } & 8 & $-2.6(0.22)^{*}$ & $-2.6(0.22)^{*}$ & $-2.4(0.21)^{*}$ & $-1.2(0.22)$ \\
\hline & 16 & $-2.9(0.23)^{*}$ & $-3.2(0.24)^{*}$ & $-2.7(0.22)^{*}$ & $-1.3(0.23)$ \\
\hline \multirow[t]{2}{*}{ COAST-W } & 8 & $-2.2(0.22)^{*}$ & $-2.2(0.20)^{*}$ & - & $-0.4(0.21)$ \\
\hline & 16 & $-2.4(0.25)^{*}$ & $-2.4(0.23)^{*}$ & - & $-0.7(0.24)$ \\
\hline \multicolumn{6}{|c|}{ Function $(\mathrm{BASFI})^{\S}$} \\
\hline \multirow[t]{2}{*}{ COAST-V } & 8 & $-2.1(0.20)^{*}$ & $-2.0(0.20)^{*}$ & $-1.9(0.19)^{*}$ & $-0.9(0.20)$ \\
\hline & 16 & $-2.4(0.22)^{*}$ & $-2.4(0.22)^{*}$ & $-2.1(0.21)^{\gamma}$ & $-1.2(0.22)$ \\
\hline
\end{tabular}

Other outcomes

Spinal pain at night

$\begin{array}{lrllll}\text { COAST-V } & 8 & -2.9(0.24)^{*} & -3.1(0.24)^{*} & -2.7(0.23)^{*} & -1.1(0.24) \\ & 16 & -3.4(0.25)^{*} & -3.6(0.26)^{*} & -2.9(0.24)^{*} & -1.6(0.25) \\ \text { COAST-W } & 8 & -2.4(0.24)^{*} & -2.3(0.22)^{*} & - & -0.5(0.23) \\ & 16 & -2.5(0.26)^{*} & -2.6(0.24)^{*} & - & -1.0(0.25)\end{array}$

Fatigue (BASDAI Q1)

\begin{tabular}{lrllll} 
COAST-V & 8 & $-2.0(0.23)^{\dagger}$ & $-2.4(0.23)^{*}$ & $-2.1(0.22)^{\gamma}$ & $-1.2(0.22)$ \\
& 16 & $-2.5(0.24)^{*}$ & $-2.6(0.24)^{*}$ & $-2.3(0.23)^{\gamma}$ & $-1.4(0.23)$ \\
COAST-W & 8 & $-1.8(0.21)^{*}$ & $-1.7(0.19)^{*}$ & - & $-0.6(0.20)$ \\
& 16 & $-2.0(0.24)^{*}$ & $-1.9(0.22)^{\gamma}$ & - & $-0.9(0.23)$ \\
\hline
\end{tabular}


Table 2 continued

\begin{tabular}{|c|c|c|c|c|c|}
\hline \multirow[t]{2}{*}{ Study } & \multicolumn{5}{|c|}{ Least-squares mean (standard error) } \\
\hline & Week & IXE Q2W & IXE Q4W & ADA & PBO \\
\hline \multicolumn{6}{|l|}{ Fatigue NRS } \\
\hline \multirow[t]{2}{*}{ COAST-V } & 8 & $-1.7(0.23)^{\dagger}$ & $-2.0(0.23)^{\gamma}$ & $-2.1(0.22)^{*}$ & $-0.9(0.22)$ \\
\hline & 16 & $-2.1(0.24)^{\dagger}$ & $-2.5(0.24)^{\gamma}$ & $-2.2(0.23)^{\dagger}$ & $-1.4(0.23)$ \\
\hline \multirow[t]{2}{*}{ COAST-W } & 8 & $-1.5(0.23)^{\gamma}$ & $-1.8(0.21)^{*}$ & - & $-0.5(0.22)$ \\
\hline & 16 & $-1.7(0.25)^{\gamma}$ & $-2.0(0.23)^{*}$ & - & $-0.7(0.24)$ \\
\hline \multicolumn{6}{|l|}{ Sleep (JSEQ) } \\
\hline \multirow[t]{2}{*}{ COAST-V } & 8 & $-2.4(0.42)^{*}$ & $-2.1(0.42)^{\gamma}$ & $-1.5(0.40)^{\dagger}$ & $-0.4(0.41)$ \\
\hline & 16 & $-3.0(0.42)^{\dagger}$ & $-2.5(0.43)$ & $-2.7(0.40)^{\dagger}$ & $-1.5(0.41)$ \\
\hline \multirow[t]{2}{*}{ COAST-W } & 8 & $-2.0(0.52)$ & $-2.6(0.47)^{\dagger}$ & - & $-0.9(0.49)$ \\
\hline & 16 & $-2.4(0.52)$ & $-3.0(0.48)$ & - & $-1.8(0.50)$ \\
\hline \multicolumn{6}{|l|}{ BASDAI $^{\S}$} \\
\hline \multirow[t]{2}{*}{ COAST-V } & 8 & $-2.3(0.21)^{*}$ & $-2.4(0.21)^{*}$ & $-2.2(0.20)^{\gamma}$ & $-1.3(0.21)$ \\
\hline & 16 & $-2.7(0.22)^{*}$ & $-2.9(0.22)^{*}$ & $-2.5(0.21)^{*}$ & $-1.4(0.22)$ \\
\hline \multicolumn{6}{|l|}{ SF-36 PCS ${ }^{\S}$} \\
\hline \multirow[t]{2}{*}{ COAST-V } & 8 & $6.4(0.74)^{*}$ & $6.5(0.75)^{*}$ & $6.2(0.71)^{*}$ & $2.0(0.73)$ \\
\hline & 16 & $8.0(0.77)^{*}$ & $7.7(0.78)^{*}$ & $6.9(0.73)^{\gamma}$ & $3.6(0.75)$ \\
\hline
\end{tabular}

$A D A$ adalimumab, $A S$ ankylosing spondylitis, $A S A S$ Assessment of Spondyloarthritis International Society, BASDAI Bath Ankylosing Spondylitis Disease Activity Index, BASFI Bath Ankylosing Spondylitis Functional Index, bDMARD biologic disease-modifying antirheumatic drugs, IXE ixekizumab, JSEQ Jenkins Sleep Evaluation Questionnaire, NRS Numeric Rating Scale, PBO placebo, $Q 2 W$ every 2 weeks, $Q 4 W$ every 4 weeks, SF-36 PCS Medical Outcomes Study 36-Item ShortForm Health Survey Physical Component Summary, TNFi tumor necrosis factor inhibitor

${ }^{*} p<0.001$ vs PBO; ${ }^{\gamma} p<0.01$ vs $\mathrm{PBO} ;{ }^{\dagger} p<0.05$ vs PBO. Comparisons were performed using a mixed-effects model for repeated measures

$\$$ BASFI, BASDAI, and SF-36 PCS data for TNFi-experienced (COAST-W) patients were published previously [13]

improvements from baseline for spinal pain at night ( 0.5 vs. $5.4, p<0.0001)$, fatigue $(0.2$ vs. $3.9, p<0.0001)$, sleep quality $(0.8$ vs. 4.9 , $p<0.0001)$, and SF-36 PCS (1.2 vs. 12.6, $p<0.0001$ ) (Fig. 4). These results corresponded to a 9.8-fold improvement in spinal pain at night, a 18.5-fold improvement for fatigue, a 5.1-fold improvement in sleep quality, and a 9.5-fold improvement in health-related QoL. Nearly identical results were observed when the analysis was conducted with only ixekizumabtreated patients (data not shown).

\section{DISCUSSION}

The primary endpoint for both the COAST-V and COAST-W clinical trials was achievement of ASAS40. Results from these studies have been published previously, showing that ASAS40 was achieved by $48-52 \%$ of bDMARD-naïve patients and $25-31 \%$ of TNFi-experienced patients who were treated with the two dosing regimens of ixekizumab, compared with $18 \%$ and $13 \%$ of patients who received placebo, respectively $[13,14]$. In the current analysis, we reported data on the impact of ixekizumab on patient- 


\section{bDMARD-naïve}
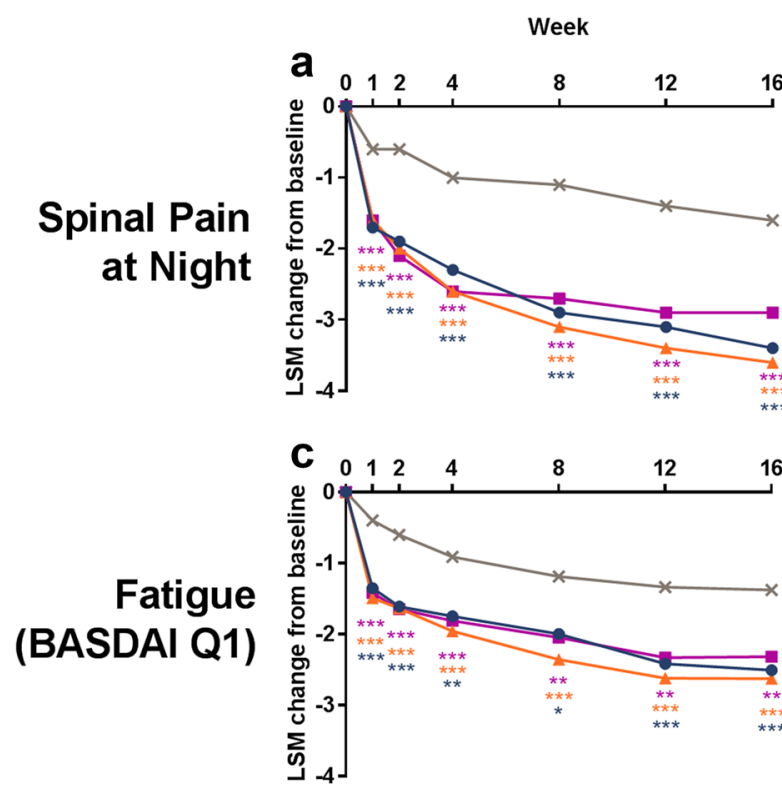

Fatigue

(NRS)

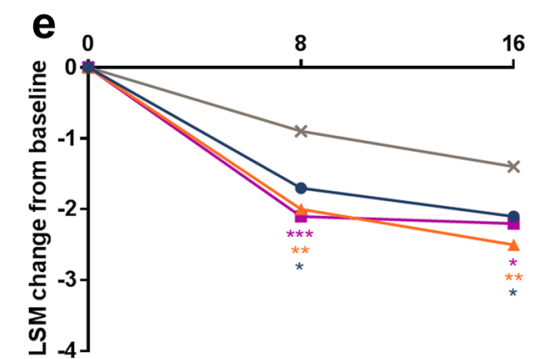

\section{Sleep quality (JSEQ)}

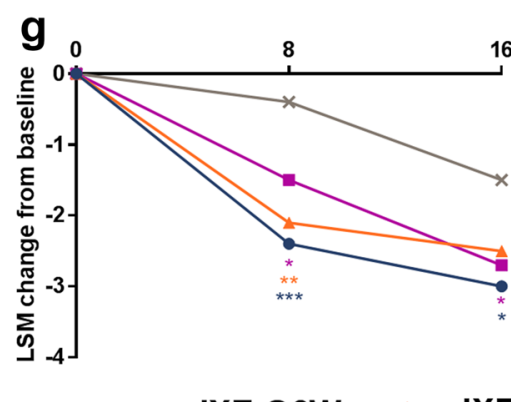

\section{TNFi-experienced}

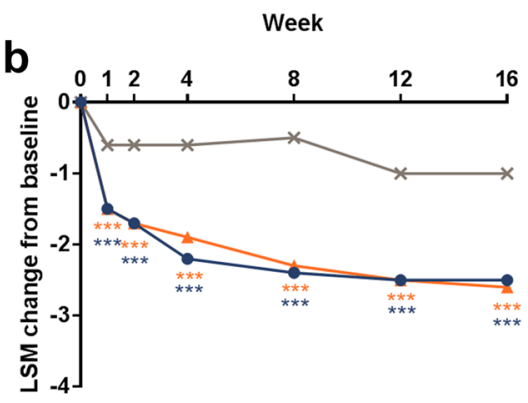

d

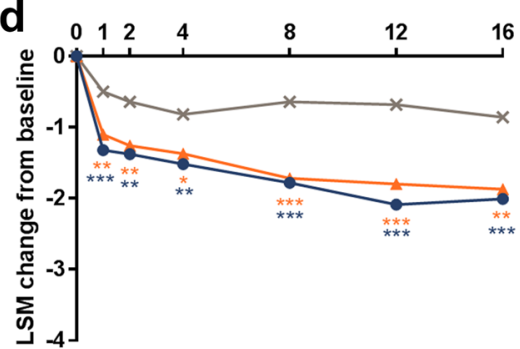

$f$

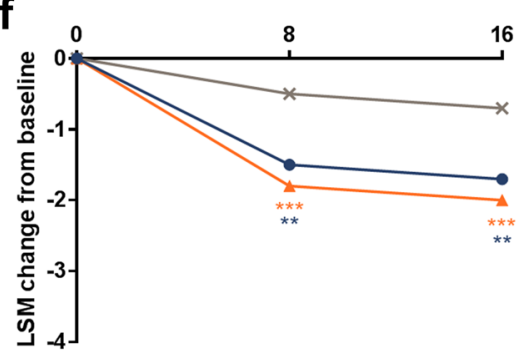

h

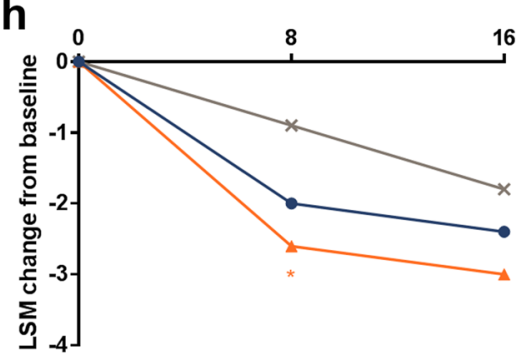

IXE Q2W

Fig. 2 Least-squares mean (LSM) changes from baseline in patient-reported outcomes of $\mathbf{a}, \mathbf{b}$ spinal pain at night, $\mathbf{c}$, d fatigue measured by BASDAI Q1, e, f fatigue measured by NRS, and $\mathbf{g}$, $\mathbf{h}$ sleep quality measured by JSEQ for $\mathbf{a}, \mathbf{c}$, e, $\mathbf{g}$ bDMARD-naïve patients (COAST-V) and $\mathbf{b}, \mathbf{d}, \mathbf{f}$, h TNFi-experienced patients (COAST-W). Comparisons with $\mathrm{PBO}$ were made using a mixed-effects model for repeated measures. ${ }^{* * *} p<0.001$ vs. $\mathrm{PBO} ;{ }^{* *} p<0.01$ vs.
$\mathrm{PBO} ;{ }^{*} p<0.05$ vs. PBO. $A D A$ adalimumab, $B A S D A I$ Bath Ankylosing Spondylitis Disease Activity Index, bDMARD biologic disease-modifying antirheumatic drugs, $I X E$ ixekizumab, JSEQ Jenkins Sleep Evaluation Questionnaire, NRS Numeric Rating Scale, $P B O$ placebo, $Q 2 W$, every 2 weeks, $Q 4 W$ every 4 weeks, $T N F i$ tumor necrosis factor inhibitor 
reported outcomes and the extent to which a better ASAS response translates to improved symptoms and health-related QoL. By translating achievement of ASAS40 into clinical measures commonly used in clinical practice, we aimed to provide physicians with scientific data that will support a better understanding of the relevance of an ASAS40 level of response from the perspective of the signs and symptoms reported by patients.

Although they have overlapping meanings, the terms "disease" and "illness" refer to slightly different aspects of patient health. Measures of disease focus on biological and chemical changes in a patient's body which are outside the normal range, while illness involves the patient's perception of this state of ill health [24]. Disease measures are very important in diagnosis and in the categorization of symptoms to inform treatment decisions in patients with AS. Although improvements in CRP levels or evidence of the resolution of sacroiliitis on imaging mark an improvement in the state of the disease, it is crucial to bear in mind that improvements in a patient's perception of their state of illness are extremely important. The data presented here show that ixekizumab treatment leads to greater improvements in patient-reported AS symptoms versus placebo treatment, including pain, fatigue, function, inflammation, sleep, and overall QoL.

The results in TNFi-experienced patients who were treated with ixekizumab for disease activity (BASDAI), function (BASFI), and health-related QoL (SF-36 PCS) have been published previously [13]. Compared to placebo, TNFiexperienced patients treated with ixekizumab

\section{bDMARD-naïve}

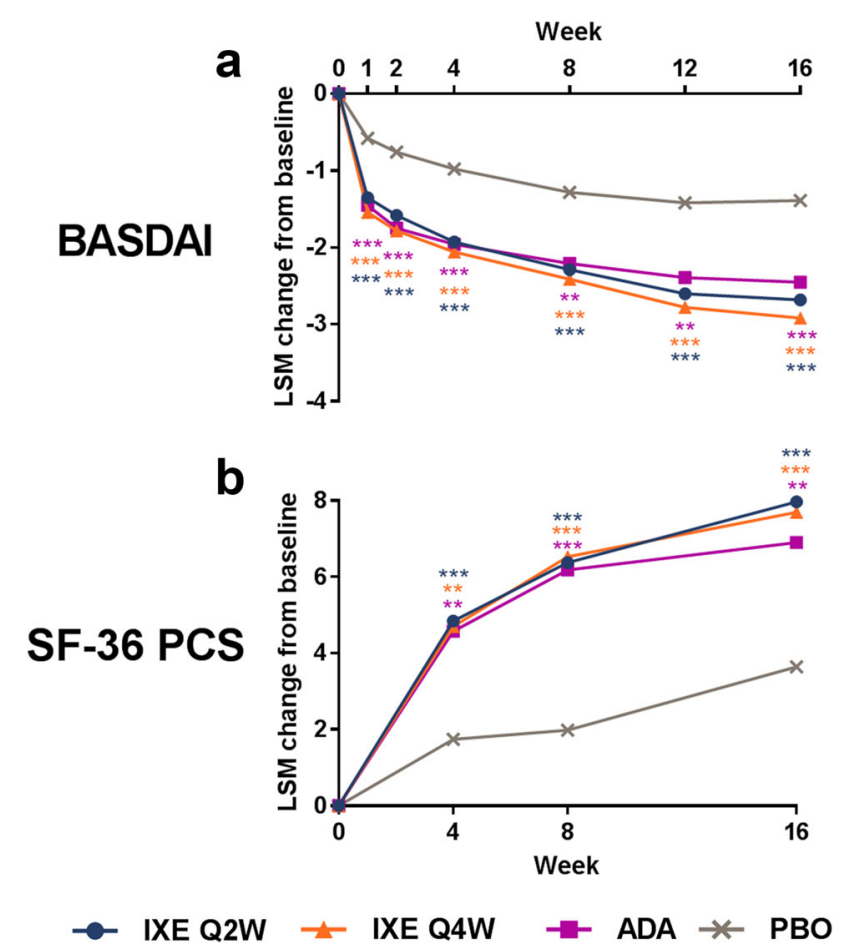

Fig. 3 Least-squares mean (LSM) changes from baseline in a Bath Ankylosing Spondylitis Disease Activity Index (BASDAI) and b Medical Outcomes Study 36-Item ShortForm Health Survey Physical Component Summary (SF36 PCS) in bDMARD-naive patients (COAST-V). Comparisons with $\mathrm{PBO}$ were made using a mixed-effects model for repeated measures. ${ }^{* * *} p<0.001$ vs. $\mathrm{PBO}$;
${ }^{* *} p<0.01$ vs. PBO. BASDAI and SF-36 PCS data for TNFi-experienced patients (COAST-W) were published previously [13]. ADA adalimumab, bDMARD biologic disease-modifying antirheumatic drug, $I X E$ ixekizumab, $P B O$ placebo, $Q 2 W$ every 2 weeks, $Q 4 W$ every 4 weeks, $T N F i$ tumor necrosis factor inhibitor 

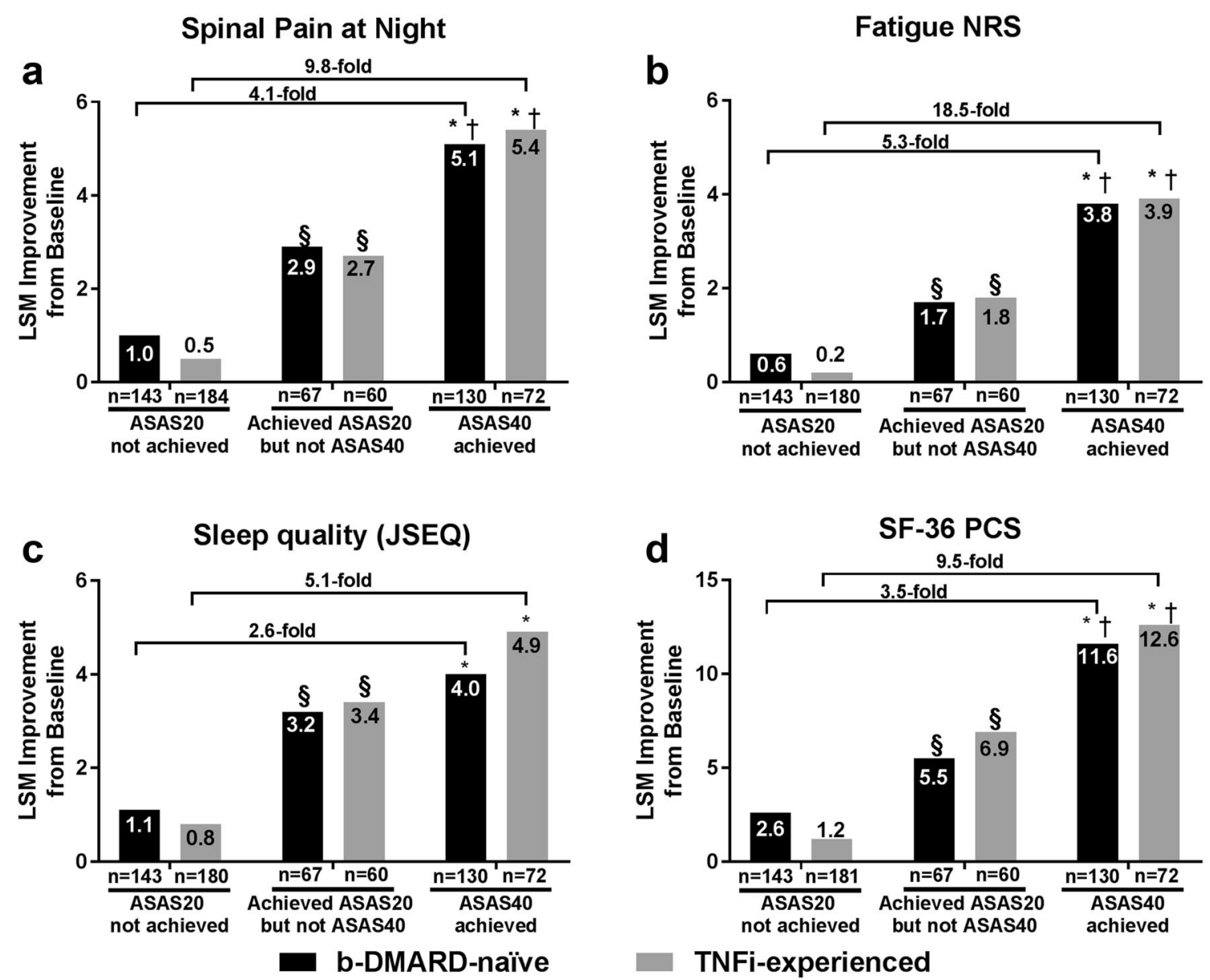

Fig. 4 Association between ASAS20 and ASAS40 and LSM improvement from baseline for a spinal pain at night, b fatigue (NRS), c sleep quality (JSEQ), and d SF-36 PCS at week 16. Treatment groups, including the placebo and adalimumab arms, were pooled and separated by ASAS20 and ASAS40 achievement. Black bars indicate data from bDMARD-naïve patients (COAST-V); gray bars indicate data from TNFi-experienced patients (COAST-W). ${ }^{\$} p$ $\leq 0.001$, achieved ASAS20 but not ASAS40 vs. ASAS20 not achieved; ${ }^{*} p<0.0001$, ASAS40 achieved vs. ASAS20 not achieved; ${ }^{\dagger} p<0.0001$, ASAS40 achieved vs. achieved ASAS20 but not ASAS40. Results were compared using ANCOVA after correcting for baseline value, age, and gender. Missing data were imputed using nonresponder

had greater levels of improvement in BASDAI, BASFI, and SF-36 PCS at each time point tested up to 16 weeks $(p<0.01)$ [13]. Because COAST$\mathrm{V}$ and COAST-W were similar in design, we were able to compare the data between the trials. It was notable that most changes from baseline for

imputation for the ASAS treatment response criteria and using modified baseline observation carried forward for the outcomes at week 16. Numbers shown are LSM improvements from baseline. Fold difference $=[$ (ASAS40 achieved/ASAS20 not achieved) - 1]. ANCOVA analysis of covariance, $A S A S$ Assessment of Spondyloarthritis International Society, $b D M A R D$ biologic disease-modifying antirheumatic drug, JSEQ Jenkins Sleep Evaluation Questionnaire, LSM least-squares mean, $n$, number of non-missing observations, NRS Numeric Rating Scale, $S F$ 36 PCS Medical Outcomes Study 36-Item Short-Form Health Survey Physical Component Summary, TNFi tumor necrosis factor inhibitor

bDMARD-naïve patients were numerically higher than those for TNFi-experienced patients, and that this was observed in both placebo- and ixekizumab-treated patients (Figs. 1, 2, 3; Table 2). By analyzing differences in response between the ixekizumab and 
placebo groups, we identified similar changes between bDMARD-naïve and TNFi-experienced patients for spinal pain (1.5 vs. 1.4-1.5), inflammation (1.6-1.9 vs. 1.7), and fatigue (1.1-1.2 vs. 1.0-1.1), respectively (Figs. 1, 2). These results are consistent with ixekizumab producing similar improvements in symptoms in both bDMARD-naïve and TNFi-experienced patients.

ASAS40 is a challenging endpoint to achieve that requires a high level of improvement in the patient's illness, as it is defined as improvements of $\geq 40 \%$ and $\geq 2$ points in three of the four domains. These four domains represent different symptoms of AS: overall disease, pain, inflammation/stiffness, and function. Although achieving ASAS40 likely represents a significant improvement in disease activity, the ASAS treatment response criteria focus on the patient's perception of improvement in symptoms and the impact that feeling better has on their ability to function. The focus on the patient's perspective and the high bar represented by ASAS40 make it a meaningful and worthy treatment goal.

Not surprisingly, the data presented here show that achieving ASAS40 equated with significant improvements in patient symptoms. The advantage of this analysis was the ability to translate these improvements into more routinely used clinical assessments. Specifically, compared with ASAS20 nonresponders, bDMARD-naïve patients who achieved ASAS40 at 16 weeks reported greater improvements in spinal pain at night (5.1-fold), fatigue (5.3-fold), sleep quality (2.6-fold), and health-related QoL (3.5-fold) versus ASAS20 nonresponders (Fig. 4). Similarly, TNFi-experienced patients who achieved ASAS40 reported greater improvements in spinal pain at night (9.8-fold), fatigue (18.5-fold), sleep quality (5.1-fold), and healthrelated QoL (9.5-fold) versus ASAS20 nonresponders (Fig. 4).

Interestingly, the fold changes in spinal pain at night, fatigue, sleep, and health-related QoL for the TNFi-experienced patients who achieved ASAS40 were all numerically greater than those for the bDMARD-naïve patients, indicating that TNFi-experienced patients felt even greater symptom relief upon achieving ASAS40. The increased responses in TNFi-experienced patients may be due to a few differences between the studies. Compared to the bDMARD-naïve patients, the TNFi-experienced patients had longer disease durations and more severe symptoms at baseline (Table 1 ). In addition, there were differences in the outcomes at week 16 for the ASAS20 nonresponders between the bDMARD-naïve patients and the TNFi-experienced patients (Fig. 4). The TNFi-experienced ASAS20 nonresponders reported smaller improvements in spinal pain at night, fatigue, and QoL compared to the bDMARD-naïve ASAS20 nonresponders (Fig. 4). Importantly, $89.9 \%$ of the TNFi-experienced patients in COAST-W had inadequately responded to one or two TNFis, but $25-31 \%$ were subsequently able to achieve ASAS40 when treated with ixekizumab. In this study, we found that many patients, even those who previously had inadequate responses to a TNFi, achieved substantial improvements in symptoms of pain, fatigue, sleep, and QoL if they attained an ASAS40 response with their treatment.

A strength of this analysis is that it included relatively high numbers of both bDMARD-naïve and TNFi-experienced patients from two randomized, placebo-controlled clinical trials, with more than 80 patients in each exposure group for both COAST-V $(n=81-90)$ and COAST-W $(n=98-114)$. While these were separate trials, the study designs for both were nearly identical, allowing comparisons to be made between the two populations. A limitation of this study is that the association analysis between ASAS treatment response and patient-reported outcomes was performed as a post hoc analysis of the COAST-V and COAST-W trials.

\section{CONCLUSION}

Patients with AS treated with ixekizumab achieved ASAS40 at significantly higher rates and had greater improvements in patient global, pain, function, inflammation/stiffness, fatigue, sleep, and health-related QoL versus patients receiving placebo. Importantly, compared with a failure to achieve ASAS20, achievement of ASAS40 was associated with a 
2.6-fold to 18.5-fold greater improvement in pain, fatigue, sleep, and health-related QoL for bDMARD-naïve and TNFi-experienced patients.

\section{ACKNOWLEDGEMENTS}

The authors thank the study participants, caregivers, and investigators.

Funding. Sponsorship for this study and article processing charges were funded by Eli Lilly and Company. All authors had full access to all of the data in this study and take complete responsibility for the integrity of the data and accuracy of the data analysis.

Medical Writing and Editorial Assistance. Emily Blue, PhD, of Eli Lilly and Company provided writing and editorial assistance. Funding for this assistance was provided by Eli Lilly and Company.

Authorship. All named authors meet the International Committee of Medical Journal Editors (ICMJE) criteria for authorship for this article, take responsibility for the integrity of the work as a whole, and have given their approval for this version to be published.

Disclosures. Philip Mease is an employee of Seattle Rheumatology Associates and has received grants and consultant and/or speaking fees from Celgene, AbbVie, Amgen, BMS, Janssen, Eli Lilly and Company, Corrona, Merck, Novartis, Crescendo, Genentech, Pfizer Inc., and UCB. Jessica Walsh is an employee of the University of Utah School of Medicine and the Salt Lake City Veterans Affairs Medical Center and has received grants and/or consultant fees from AbbVie, Celgene, Novartis, and Eli Lilly and Company. Xenofon Baraliakos is an employee of Rheumazentrum Ruhrgebiet Herne and has received grants, research support, or consultation fees from AbbVie, BMS, Celgene, Chugai, Eli Lilly, Galapagos, Janssen, MSD, Novartis, Pfizer, and UCB. Robert Inman is an employee of University Health Network and received grants, personal fees, and/or honoraria from AbbVie, Amgen, Janssen, Eli Lilly and Company, Merck, and Novartis. Kurt de Vlam is an employee of University Hospitals Leuven and KU Leuven and has received speaker's and consultancy fees from AbbVie, Celgene, Eli Lilly and Company, Johnson \& Johnson, Merck, Novartis, Pfizer, and UCB. James Cheng-Chung Wei is an employee of Taiwan TTY and has received consultant and/or speaking fees from Johnson \& Johnson, AbbVie, UCB, and Novartis. Theresa Hunter is an employee of and owns stock in Eli Lilly and Company. Gaia Gallo is an employee of and owns stock in Eli Lilly and Company. David Sandoval is an employee of and owns stock in Eli Lilly and Company. Fangyi Zhao is an employee of and owns stock in Eli Lilly and Company. Yan Dong is an employee of and owns stock in Eli Lilly and Company. Rebecca Bolce is an employee of and owns stock in Eli Lilly and Company. Helena Marzo-Ortega is an employee of Leeds Teaching Hospitals Trust and Leeds Institute of Rheumatic and Musculoskeletal Medicine and has received personal fees, grant or research support, consultant fees, or speaker fees for AbbVie, Celgene, Janssen, Eli Lilly and Company, Novartis, Pfizer, and UCB.

Compliance with Ethics Guidelines. All procedures performed in these studies involving human participants were in accordance with the ethical standards of the institutional and/or national research committees at all sites where these studies were conducted and with the 1964 Declaration of Helsinki and its later amendments or comparable ethical standards. The master ethics committee was Schulman Associates IRB, Cincinnati, OH, USA. The full lists of investigators and sites are provided in the primary manuscript supplements [13, 14]. Informed consent was obtained from all individual participants included in the studies.

Data Availability. The datasets generated and/or analyzed during the current study are available from the corresponding author on reasonable request.

Open Access. This article is distributed under the terms of the Creative Commons 
Attribution-NonCommercial 4.0 International License (http://creativecommons.org/licenses/ by-nc/4.0/), which permits any noncommercial use, distribution, and reproduction in any medium, provided you give appropriate credit to the original author(s) and the source, provide a link to the Creative Commons license, and indicate if changes were made.

\section{REFERENCES}

1. Reveille JD. Epidemiology of spondyloarthritis in North America. Am J Med Sci. 2011;341(4):284-6.

2. Gran JT, Husby G, Hordvik M. Prevalence of ankylosing spondylitis in males and females in a young middle-aged population of Tromso, Northern Norway. Ann Rheum Dis. 1985;44(6):359-67.

3. Rudwaleit M, van der Heijde D, Landewe R, et al. The development of Assessment of SpondyloArthritis international Society classification criteria for axial spondyloarthritis (part II): validation and final selection. Ann Rheum Dis. 2009;68(6):777-83.

4. Strand V, Singh JA. Patient burden of axial spondyloarthritis. J Clin Rheumatol. 2017;23(7):383-91.

5. van der Heijde D, Ramiro S, Landewe R, et al. 2016 Update of the ASAS-EULAR management recommendations for axial spondyloarthritis. Ann Rheum Dis. 2017;76(6):978-91.

6. Glintborg B, Ostergaard M, Krogh NS, Dreyer L, Kristensen HL, Hetland ML. Predictors of treatment response and drug continuation in 842 patients with ankylosing spondylitis treated with anti-tumour necrosis factor: results from 8 years' surveillance in the Danish nationwide DANBIO registry. Ann Rheum Dis. 2010;69(11):2002-8.

7. Heiberg MS, Koldingsnes W, Mikkelsen K, et al. The comparative one-year performance of anti-tumor necrosis factor alpha drugs in patients with rheumatoid arthritis, psoriatic arthritis, and ankylosing spondylitis: results from a longitudinal, observational, multicenter study. Arthritis Rheum. 2008;59(2):234-40.

8. Inman RD, Davis JC Jr, Heijde D, et al. Efficacy and safety of golimumab in patients with ankylosing spondylitis: results of a randomized, double-blind, placebo-controlled, phase III trial. Arthritis Rheum. 2008;58(11):3402-12.
9. van der Heijde D, Kivitz A, Schiff MH, et al. Efficacy and safety of adalimumab in patients with ankylosing spondylitis: results of a multicenter, randomized, double-blind, placebo-controlled trial. Arthritis Rheum. 2006;54(7):2136-46.

10. Dougados M, Baeten D. Spondyloarthritis. Lancet. 2011;377(9783):2127-37.

11. Yeremenko N, Paramarta JE, Baeten D. The interleukin-23/interleukin-17 immune axis as a promising new target in the treatment of spondyloarthritis. Curr Opin Rheumatol. 2014;26(4):361-70.

12. Liu L, Lu J, Allan BW, et al. Generation and characterization of ixekizumab, a humanized monoclonal antibody that neutralizes interleukin-17A. J Inflamm Res. 2016;9:39-50.

13. Deodhar A, Poddubnyy D, Pacheco-Tena C, et al. Efficacy and safety of ixekizumab in the treatment of radiographic axial spondyloarthritis: sixteenweek results of a phase III randomized, doubleblind, placebo-controlled trial in patients with prior inadequate response or intolerance to tumor necrosis factor inhibitors. Arthritis Rheumatol. 2019;71(4):599-611.

14. van der Heijde D, Cheng-Chung Wei J, Dougados $\mathrm{M}$, et al. Ixekizumab, an interleukin-17A antagonist in the treatment of ankylosing spondylitis or radiographic axial spondyloarthritis in patients previously untreated with biological disease-modifying anti-rheumatic drugs (COAST-V): 16 week results of a phase 3 randomised, double-blind, active-controlled and placebo-controlled trial. Lancet. 2018;392(10163):2441-51.

15. Sieper J, Rudwaleit $M$, Baraliakos $X$, et al. The Assessment of SpondyloArthritis international Society (ASAS) handbook: a guide to assess spondyloarthritis. Ann Rheum Dis. 2009;68(Suppl 2):ii1-44.v.

16. Baeten D, Sieper J, Braun J, et al. Secukinumab, an interleukin-17A inhibitor, in ankylosing spondylitis. N Engl J Med. 2015;373(26):2534-48.

17. Davis JC, van der Heijde DM, Braun J, et al. Sustained durability and tolerability of etanercept in ankylosing spondylitis for 96 weeks. Ann Rheum Dis. 2005;64(11):1557-62.

18. Lambert RG, Salonen D, Rahman P, et al. Adalimumab significantly reduces both spinal and sacroiliac joint inflammation in patients with ankylosing spondylitis: a multicenter, randomized, double-blind, placebo-controlled study. Arthritis Rheum. 2007;56(12):4005-14. 
19. van der Heijde D, Dougados M, Landewe R, et al. Sustained efficacy, safety and patient-reported outcomes of certolizumab pegol in axial spondyloarthritis: 4-year outcomes from RAPID-axSpA. Rheumatology (Oxford). 2017;56(9):1498-509.

20. van der Linden S, Valkenburg HA, Cats A. Evaluation of diagnostic criteria for ankylosing spondylitis. A proposal for modification of the New York criteria. Arthritis Rheum. 1984;27(4):361-8.

21. Machado PM, Landewé R, van der Heijde D. Ankylosing Spondylitis Disease Activity Score (ASDAS): 2018 update of the nomenclature for disease activity states. Ann Rheum Dis. 2018;77:1539-40.
22. Naegeli AN, Flood E, Tucker J, Devlen J, EdsonHeredia E. The patient experience with fatigue and content validity of a measure to assess fatigue severity: qualitative research in patients with ankylosing spondylitis (AS). Health Qual Life Outcomes. 2013;11:192.

23. Deodhar A, Braun J, Inman RD, et al. Golimumab reduces sleep disturbance in patients with active ankylosing spondylitis: results from a randomized, placebo-controlled trial. Arthritis Care Res (Hoboken). 2010;62(9):1266-71.

24. Helman CG. Disease versus illness in general practice. J R Coll Gen Pract. 1981;31(230):548-52. 